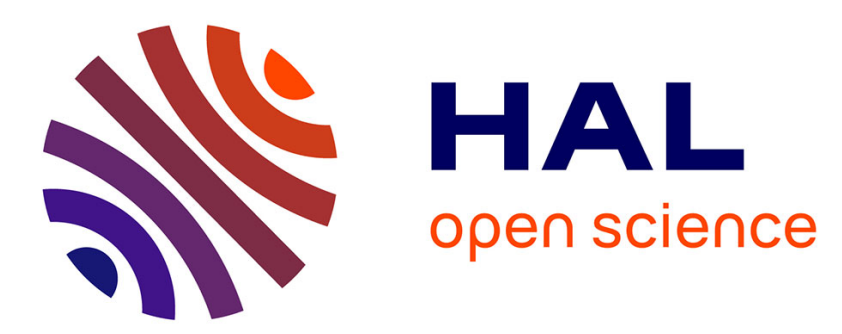

\title{
Les valeurs écoresponsables des expéditions en fauteuil tout terrain, un moyen de renforcer la mise en scène de la différence handi-capable
}

Eric Perera, Roland Thaler, Andre Galy

\section{- To cite this version:}

Eric Perera, Roland Thaler, Andre Galy. Les valeurs écoresponsables des expéditions en fauteuil tout terrain, un moyen de renforcer la mise en scène de la différence handi-capable. STAPS : Revue internationale des sciences du sport et de l'éducation physique, 2021. hal-03258977

\section{HAL Id: hal-03258977 \\ https://hal.umontpellier.fr/hal-03258977}

Submitted on 12 Jun 2021

HAL is a multi-disciplinary open access archive for the deposit and dissemination of scientific research documents, whether they are published or not. The documents may come from teaching and research institutions in France or abroad, or from public or private research centers.
L'archive ouverte pluridisciplinaire $\mathbf{H A L}$, est destinée au dépôt et à la diffusion de documents scientifiques de niveau recherche, publiés ou non, émanant des établissements d'enseignement et de recherche français ou étrangers, des laboratoires publics ou privés. 
Les valeurs éco-responsables des expéditions en fauteuil tout terrain, un moyen de renforcer la mise en scène de la différence handi-capable.

\section{The eco-friendly values of expeditions in all-terrain wheelchairs, a means to strengthen the visibility of the handi-capable difference.}

ERIC PERERA, ROLAND THALER, ANDRE GALY

ERIC PERERA, MCU HDR - UFR STAPS Université de Montpellier (SantESiH, EA n4614), eric.perera@umontpellier.fr T. 0411759091

ROLAND THALER, Dirigeant Agence Altitude, Groupe Ecovia, Enseignant Département Biologie Ecologie, Université de Montpellier, roland.thaler@umontpellier.fr

ANDRE GALY, PAST - UFR STAPS Université de Montpellier (SantESiH, EA n4614) Coach professionnel, consultant tourisme et loisirs outdoor - Tendance Montagne andre.galy@umontpellier.fr

RÉSUMÉ: L'usage de Fauteuils Tout Terrain (FTT) permet un accès au milieu naturel des personnes handicapées. Cette pratique est révélatrice d'expériences sociales et de sensations de proximité avec la nature. Plus remarquable encore, les expéditions réalisées en FTT supposent des logiques slow en intégrant des préoccupations éthiques et environnementales contemporaines. Effectivement, l'esprit du FTT, impulsé par les pionniers de cette pratique en France dès 1990, persiste et consiste à prévoir des adaptations pour accéder à la nature et non l'inverse.

Cependant l'impact carbone des expéditions lointaines en FTT interroge sur l'affichage d'une prise en compte écologique. Cet impact est d'autant plus sujet à débat que la nouvelle génération d'handisportifs promeut l'handicapacité en recherchant une autonomie la plus complète possible. Assurément, l'étude du montage d'un projet d'expédition en FTT (Chili), montre que le développement de FTT innovants à maindalier avec assistance électrique tend à reléguer la préoccupation éco-responsable au second plan. Seulement, la recherche de performance n'éloigne pas pour autant d'une approche écoresponsable car il est nécessaire de penser un usage minimal des aides modernes, et ainsi d'un moindre impact. Ces expéditions FTT, qui sont par nature impactantes en moyens de transports transcontinentaux, impliquent un équilibre entre engagement handi-capable et affichage écoresponsable.

MOTS CLÉS : FTT, expédition, handisport, écoresponsable, innovation.

\footnotetext{
ABSTRACT : Using an All-Terrain Wheelchair (ATW) allows disabled people to gain access to natural spaces. This activity provides social experiences and feelings of proximity to nature. Even more remarkable, expeditions carried out using ATW follow slow rules by integrating contemporary ethical and ecological preoccupations. Indeed, the ATW spirit, generated by the pioneers of this practice in France in the 1990s, still remains and consists in creating adaptations in order to access nature, and not the opposite.
} 
However, the carbon footprint of distant expeditions using ATWs questions the image of an eco-friendly outlook. This footprint is all the more a subject for debate that the new generation of handiathletes promote handicapacity in their search for the most complete autonomy possible. Indeed, studying the organisation of an ATW expedition (Chile) revealed that developing innovating ATWs using electrically assisted hand-cranks tends to relegate eco-friendly concerns to the second place. However, the search for performance does not necessarily deviate from an eco-friendly approach, since it implies seeking a minimal use of modern assistance means, and thus having a smaller impact. These ATW expeditions, which by nature have an impact in terms of transcontinental transport means, imply a balance between handi-capable engagement and eco-friendly designs.

KEY WORDS: ATW, expedition, handisport, eco-friendly, innovation. 


\title{
Les valeurs éco-responsables des expéditions en fauteuil tout terrain, un moyen de renforcer la mise en scène de la différence handi-capable.
}

\author{
ERIC PERERA, ROLAND THALER, ANDRE GALY
}

\section{INTRODUCTION}

Depuis quelques années l'aventure en milieu naturel devient possible pour les personnes handicapées (Pantaléon et Reichhart, 2017) grâce à l'usage d'engins de plus en plus perfectionnés, dont le Fauteuil Tout Terrain (FTT) (Villoing et al., 2017). Le FTT procure une expérience sociale (Issanchou \& Perera, 2020) et des sensations inédites (Perera et al., 2017) proches du VTT. Il offre aussi la possibilité de mixité (valides et handisportifs).

Le FTT semble a priori relever du «tourisme contemplatif » instaurant « une sensorialité intimiste avec la nature et la culture » (Bourdeau, 1994). Il se rapprocherait de préoccupations éthiques et environnementales contemporaines, s'apparentant au slow tourisme (colloque Slow tourisme/Slow sport 2018 - Université Littoral Côte d'Opale) intégrant les mouvements du slow sport en se fondant dans la nature par une cosmose (Andrieu, 2017). L'empreinte du touriste y est minimisée, ses comportements étant pensés pour rentrer en communion avec ce qu'il observe (Corneloup, 2011).

Cependant la dimension écologique des expéditions FTT interroge, notamment quant à l'impact carbone des déplacements ou encore l'usage d'engins motorisés pour la logistique. Par ailleurs, de nouveaux FTT font appel à l'assistance électrique pour parcourir la montagne en autonomie (Perera et al., 2020). Dans quelle mesure l'affichage d'une prise en compte écologique est-elle alors envisageable pour dépasser l'idée de simplement « verdir » (Marsac et $a l ., 2012$ ) l'image d'une expédition lointaine en FTT ?

La question se pose d'autant que la nouvelle génération des sportifs en FTT promeut l'handicapacité en recherchant une autonomie la plus complète possible. Ces nouvelles approches permettent de développer des FTT innovants à maindalier avec assistance électrique pouvant reléguer la préoccupation éco-responsable au second plan.

\section{MÉTHODOLOGIE}

Nous nous intéresserons à la dimension écologique des expéditions FTT à partir d'entretiens de type «histoire de vie » (Bertaux, 1997), proposant un regard socio-historique sur leur organisation (entretiens avec Jean-François Porret (JFP) et Gilles Bouchet (GB), pionniers de l'activité FTT). L'étude de documents concernant l'équipement d'expédition apportera des informations complémentaires. Des entretiens ont été aussi réalisés avec Gilbert Marmey (GM), concepteur d'un FTT électrique, et Vincent Bourry (VB), pratiquant régulier impliqué dans un projet innovant de FTT électrique. Une enquête ethnographique consacrée au suivi d'un projet d'expédition FTT en Bolivie débuté en mars 2018 complète ces données. L'objectif de l'expédition est double : réaliser un parcours intégrant l'ascension de l'Uturuncu (6008 mètres), et porter une image positive du handicap (ou du « capable autrement » selon le parrain de l'expédition Philippe Croizon, célèbre nageur amputé des 4 membres). La COVID 19 a différé le projet à une date non fixée à ce jour. Les données produites in situ (Perera et 
Beldame, 2016) par les chercheurs, auteurs du présent article et accompagnateurs actifs, concernent à ce jour des observations sur la préparation logistique de l'expédition et les entraînements. Cela a permis d'observer les processus sociaux sous-jacents de l'engagement de six personnes avec handicaps mixtes utilisant des engins adaptés et neuf accompagnateurs. On constate alors que la valorisation de l'expédition privilégie l'autonomie des handisportifs et s'éloigne a priori de logiques éco-responsables. En effet, la sportivité attendue, gage d'une mise en scène handicapable, implique l'usage d'une assistance électrique et surtout, une logistique imposante ( $4 \times 4$, groupes électrogènes, etc.). Ceci ne permet pas en l'état un affichage écologique suffisamment performant, malgré la réelle volonté de tous de limiter les impacts. Mais cela permet en revanche de définir les pistes de progrès pour parvenir à une « écoresponsabilisation » du groupe dans la préparation et l'exécution de l'expédition (usage des véhicules optimisé, parcours condensés, couchage sous tente...).

\section{L'EMERGENCE DU FTT EN FRANCE, DES VALEURS DE PARTAGE ET DE PROXIMITE AVEC LA NATURE}

La pratique du FTT émerge en France en 1990 par l'intermédiaire de JFP, alpiniste français reconnu et tétraplégique suite à un accident de parapente en 1989. En rééducation, il découvre le "Cobra", premier FTT inspiré de la technologie «mountain bike ». Fort de son expérience de la montagne, il réalisera une première expédition au Kula Kangri (Tibet) en 1993, atteignant l'altitude de 5600m. Il retrouve ainsi «ce plaisir de monter des projets, de rêver de quelque chose [...], de retrouver l'incertitude de la réussite [...] c'était vraiment une composante forte de continuer la vie ». Avec l'aide de GB, ils testent une centaine de parcours, essentiellement dans les Alpes (www.ftt.free.fr). Cette pratique du FTT est volontairement «tournée vers la randonnée sportive et la recherche de l'autonomie en pleine nature » (HandiMag, Porret, 1999, p. 41). La fin des années 2000 voit se développer une offre de FTT enfin accessible (Villoing et al., 2017). Que ce soit par la randonnée ou les expéditions, ce pionnier du FTT retrouve l'accès à la montagne, et aux valeurs de partage et de mixité handicapés/valides. Ces valeurs de partage tout comme celle qui consiste à instaurer une certaine proximité avec la nature, sont au fondement des logiques de la pratique du FTT et la rapprochent, dans son activité du slow sport et dans sa conception du voyage, du slow tourisme.

\subsection{La pratique du FTT : «la montagne du partage » avant la performance}

Les expéditions de JFP sont avant tout des expériences sociales jouant un rôle essentiel dans la continuité de sa pratique de l'alpinisme, passant d'une logique de performance à une logique de partage. «Il y avait quand même avant (l'accident) la montagne de la performance et après la montagne du partage [...] ça ne veut pas dire qu'il n'y a pas de partage avant et qu'il n'y a pas de performance après ». C'est surtout grâce à cette transition vers une logique de partage qu'il va relancer «les expéditions comme avant». Mais en faisant «en sorte que ceux qui viennent avec moi aient leur propre plaisir [...]. Moi j'ai forcément le but de [...] retrouver les sensations d'expédition sur mes jambes [...] (avec) un niveau d'inconfort qui m'oblige à prendre sur moi comme je le faisais avant, un niveau d'inconfort qui est finalement assez semblable... ». JFP note dans son carnet de bord l'importance du groupe : l'aventure «n'aurait pas été possible sans la constante sollicitude de toute l'équipe et sa capacité d'adaptation aux contraintes apportées par le FTT (expédition 
Minya Kongka, Chine, 1995). Il met ainsi en évidence les effets de la mixité "handis" et valides qui permettent de vivre une expérience sociale forte.

De plus, sa condition de tétraplégique va produire un autre rapport aux populations locales lors des expéditions. Ainsi, le FTT offre une occasion de mieux se rapprocher de populations qui lui apportent «tellement de gentillesse dans toutes ces belles rencontres» (carnet Minya Kongka, 1995). Ainsi, les expéditions de JFP ont évolué en «aventure non pas au sens aventurier mais au sens contact avec les gens, sentir cette chaleur humaine extraordinaire.» Il s'agit d'une autre manière d'aborder les expéditions prônant «la montagne partage par rapport à la montagne performance ».

JFP privilégie le lien social (équipiers, populations locales), la logique de partage prenant le dessus sur la recherche de performance. La pratique du FTT permet dès lors de découvrir sportivement des espaces naturels en compagnie de pairs (Sirost, 2010) générant un rapport à l'environnement différent.

\subsection{La pratique du FTT, une activité respectueuse de la montagne}

L'environnement joue un rôle central dans les expéditions FTT. JFP cherchait autrefois l'exploit : à ouvrir une nouvelle voie au Pérou, accéder à un sommet vierge au Pakistan.... Après son accident, il continue à s'aventurer dans des lieux lointains et sauvages, ses sensations semblant décuplées par sa nouvelle condition: "Le temps se dégage et nous offre une superbe vue sur l'ensemble du massif du Kula Kangri. Je vois se concrétiser sous mes yeux ce que j'avais imaginé depuis les photos en 87 [...] Nous voyons tout l'itinéraire de la voie japonaise et nous dominons nettement le glacier d'accès de l'alpinisme vu en fauteuil roulant... [...]. Instants de bonheur trop rares, que ces minutes de totale liberté intensément vécues à l'autre bout du monde ». Cette approche s'inscrit dans un profond respect pour la montagne et prend de l'importance suite à son accident : "La nature on n'y touche pas [...] on prévoit les adaptations qui permettent au handicap d'accéder à cette nature pas touchée. Plutôt que de mettre la nature à la portée du handicap, c'est mettre le handicap à la portée de la nature ».

Le FTT est conçu pour faciliter l'accès aux randonnées de pleine nature. Pour JFP, l'usage de cet engin, entre "dépassement des limites » et «bonheur de l'autonomie » permet de démontrer que le handicap n'est pas un obstacle à l'aventure. Que ce soit avant ou après son accident les moyens mis en place restent les mêmes (avions, véhicule, porteurs/animaux). Sa tétraplégie l'oblige cependant à se déplacer avec son FTT, son fauteuil standard, et beaucoup de matériel spécifique à sa condition. Ceci occasionnant un surpoids non négligeable comparé aux expéditions «normales». Il doit de plus faire appel à une aide extérieure pour réaliser l'ascension du sommet (porteurs supplémentaires, et surtout tractation par des animaux...).

Hors ce minimum, l'importance de l'environnement est telle pour JFP, qu'il considère que c'est le handicap qui doit s'adapter à la montagne. Le FTT représente un véritable instrument sportif (Richard, 2017) pour la haute montagne qui permet à JFP d'accomplir ses nombreuses expéditions.

\section{REPRESENTATIONS ET LEVIERS D'UNE EXPEDITION DURABLE EN FTT}

Pionniers du FTT en France, JFP et GB ouvrent la voie aux possibilités d'accès aux endroits naturels les plus extrêmes. On assiste alors à un développement de la pratique, largement facilité par la Fédération Française de Handisport $(\mathrm{FFH})^{\mathrm{i}}$ pour répondre à l'engouement du public pour les sports de nature. Ainsi, la FFH créera dès $1998^{\mathrm{ii}}$ diverses manifestations, telle que «l'X Trême Day handisport» en Aveyron par exemple. Au niveau 
fédéral, les sports d'aventure se développent en mettant l'accent sur les expéditions. La FFH «s'approprie » ainsi ces activités qui deviennent un complément de la dimension compétitive qui dominait jusqu'alors. Les financements apportés aux expéditions et raids ${ }^{\mathrm{iii}}$, vont profiter au développement de l'activité FTT en général.

En parallèle, la recherche d'autonomie pour surmonter les barrières naturelles mais aussi sociales, génère le développement des FTT avec assistance électrique. Avec le FTT électrique, "on change de paradigme » pour atteindre "l'autonomie complète » d'après Vincent Boury (Le Roux et al., 2018). Si l'autonomie est valable tant pour la descente que pour la montée, cette nouvelle approche chamboule les interactions valides/handicapés dans la pratique du FTT classique et ce d'autant plus, que la nouvelle génération souhaite un engagement encore plus sportif. Cette technologie est pensée avant tout comme un engagement sportif autonome et s'éloigne ainsi d'une logique durable.

\subsection{L'assistance électrique, une aide pour la sportivité en pleine nature}

Les premiers modèles de FTT à assistance électrique émergent en 2006, permettant de descendre les chemins montagneux mais aussi et surtout de faciliter les ascensions sans aucune aide. Avec la Coccinelle ${ }^{\text {iv }}$, développée par l'association Coccinnelle-Handi et le Boma $^{v}$, industrialisé par la société anglaise Molten Rock, ces engins marquent une évolution dans la pratique du FTT, permettant une autonomie croissante de la personne handicapée.

D'autres modèles de FTT AE comme le Mobile Dream ${ }^{\text {vi }}$, le e-buggy ${ }^{\text {vii }}$ ou encore le Quadrix ${ }^{\text {viii }}$ arrivent sur le marché, à la fois robustes et dotés de grandes capacités d'autonomie grâce à l'évolution des batteries. Toutefois, ces FTT AE sont plutôt pensés pour la «balade » (VB) et non pour des performances sportives ou des expéditions tel que le permet le FTT classique qui peut se bricoler facilement comme l'a fait par exemple le chef d'expédition, Gérard Genthon $(\mathrm{GG})^{\mathrm{ix}}$. Dans ce sens, le FTT AE ouvre de nouvelles manières de pratiquer la pleine nature centrée sur une pratique loisir familiale et non la performance.

Pourtant c'est bien le FTT AE qui est choisi par la nouvelle génération d'handisportifs dans le cadre de l'expédition Uturuncu : choix de la vitesse et de la participation physique, contre l'esprit «balade », qui va entrainer un projet de nouveau type de FTT avec maindalier à assistance électrique ${ }^{\mathrm{x}}$, permettant au FTTiste d'être totalement partie prenante de son activité.

Les valeurs portées par une partie de l'équipe tant handisportifs que valides privilégiant plus d'autonomie et de sportivité, entrainent une rupture qui s'accompagne du changement de chef d'expédition, de GG à Emmanuel Senin, de l'ancienne génération à la nouvelle. L'équipe recomposée se réunira le 9 août 2019 pour réorienter l'expédition autour des enjeux et des considérations des handisportifs impliquant un nouvel engin FTT à maindalier AE mais aussi un parcours d'expédition plus sportif. Le choix de mettre en scène l'handisportif acteur de l'effort durant l'ascension passe par un usage de technologies ajustées et un parcours qui présente une dimension sportive et de prise de risque. Cependant cette logique d'autonomie sportive différente de l'esprit de l'expédition initiale (appelée Handi'Cap Cordillère), n'empêche pas d'entretenir des valeurs de partage handisportifs/valides et le maintien d'une certaine intimité avec la nature.

\subsection{Un positionnement durable de l'expédition FTT}

Les valeurs communes de l'expédition Handi'Cap Cordillère ont été débattues lors du week-end d'entraînement dans le massif du Lubéron le 6 juin 2019. L'enjeu étant de penser collectivement l'image que l'équipe voulait donner à l'expédition, ceci afin de présenter un discours homogène vis-à-vis des parties prenantes. Ce temps d'échanges s'est avéré nécessaire 
car la vision de l'expédition Handi'Cap Cordillère semblait différer nettement selon les membres de l'équipe. Il en allait d'une part de la cohésion du groupe, d'autre part de la cohérence de la communication pour la recherche de partenariats et sponsors. Que doit-on mettre en avant collectivement, reflétant l'esprit et les valeurs de l'expédition ? Tel était le questionnement posé. Ce débat a été riche et déterminant pour le devenir du projet.

Emmanuel a ainsi mis avant l'importance des valeurs sportives et humanitaires vis-àvis des mécènes sollicités : "J'avais du mal à m'identifier sur le support (en parlant de plaquette préexistante). [...] je pense que ça serait intéressant de revoir ce qu'on met en avant: le côté exploit, le 6000... [...] si on choisit les fondations... Je parlerais aussi d'humanitaire [...] c'est un plus sur place pour les gens que l'on peut aider et ça nous ouvre plus de portes».

Le choix de ces valeurs, approuvées par l'équipe, et la difficulté pour Emmanuel de s'identifier dans la communication alors en cours, ont entraîné un changement d'orientation de l'expédition. En effet, les valeurs «slow » portées jusqu'alors notamment par GG étaient peu compatibles avec la mise en scène de la performance et de l'autonomie des handisportifs, sous prétexte d'une solidarité handicapés/valides autour d'un FTT classique (Perera et al., 2020).

Il en découlera un remaniement de l'équipe et l'émergence d'un projet renouvelé de l'expédition, désormais intitulé Uturuncu. Ce projet marque une volonté de valoriser avant tout l'handicapable, tout en intégrant des principes écoresponsables. Ce sujet avait en effet déjà largement fait débat, par exemple concernant l'usage de véhicules sur place : " [...] dans le projet initial, il y a neuf 4X4 ! [...] Est-ce qu'on n'est pas alors plus dans le confort que dans l'expédition? Ce qui vient percuter le sens écoresponsable. Est-ce qu'on ne peut pas se serrer et mettre des choses sur le toit, et alors on en a plus que 3 ?». Autre sujet discuté : l'alimentation : "Manger local ça parait une évidence, mais c'est compliqué, qu'est-ce qu'on mange ? [...] Peut-être qu'il y a un équilibre à discuter ». Au final se révèle le besoin de définir collectivement les axes d'écoresponsabilités pour l'expédition : "On n'a pas défini entre nous les leviers pour rendre le projet responsable et du coup pour être crédible pour en parler. »(Julien).

La recherche d'un équilibre entre approche sportive et approche écoresponsable va donc guider la réflexion et aider à déterminer non seulement le parcours du nouveau projet Uturuncu mais aussi la conception du nouveau modèle de FTT à maindalier AE à tester.

UNE EXPÉRIENCE AU BÉNÉFICE DE TOUS

Au-delà de la performance sportive, notre expédition revêt des enjeux sociétaux d'aujourd'hui et de demain : promotion de la solidarité, partage de savoirs et savoir-faire avec les acteurs locaux, développement de l'accessibilité aux sports et à la nature pour tous.

tels sont les retours d'expérience attendus de cette aventure collective.

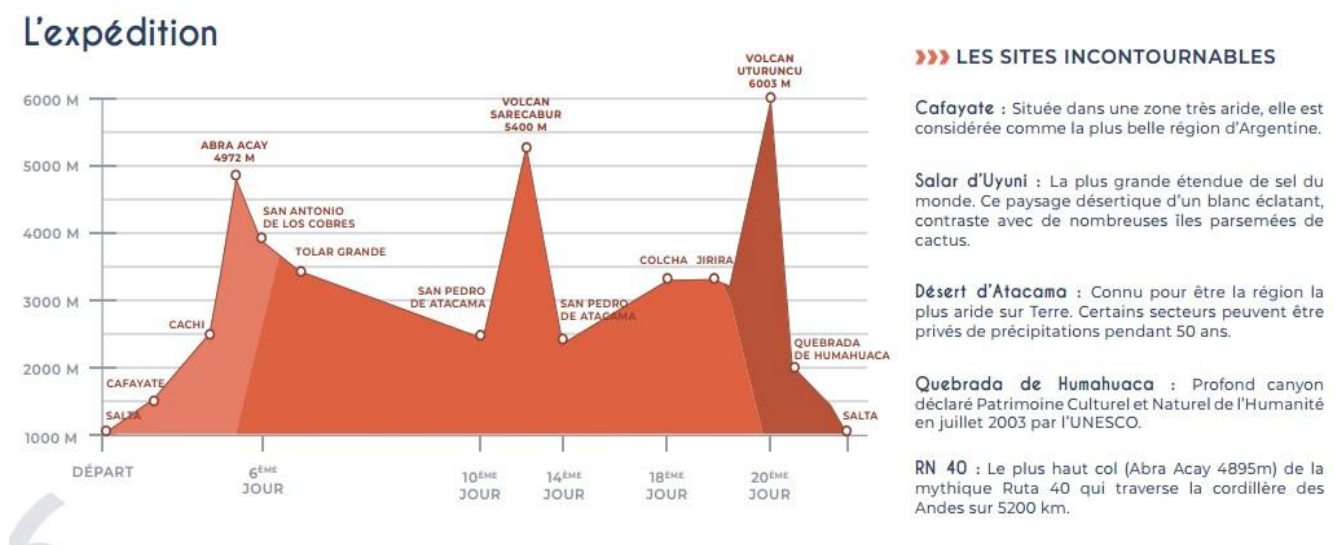

Plaquette Expédition Uturuncu - page 6 
La nouvelle plaquette de promotion souligne les dénivelés auxquels les membres de l'équipe vont devoir se confronter et l'effort des handisportifs à réaliser ces ascensions en autonomie. Ces nouveaux défis permettent de développer des engins innovants, tel qu'un FTT à maindalier incluant l'assistance électrique (cf. image ci-dessous), pour un engagement autonome et plus sportif. Mais si les sommets à atteindre, dont l'Uturuncu et ses $6003 \mathrm{~m}$, sont mis en avant, le parcours pointe également les «sites incontournables » qui seront traversés. La dimension contemplative du séjour demeure donc, malgré une sportivité du parcours accrue.
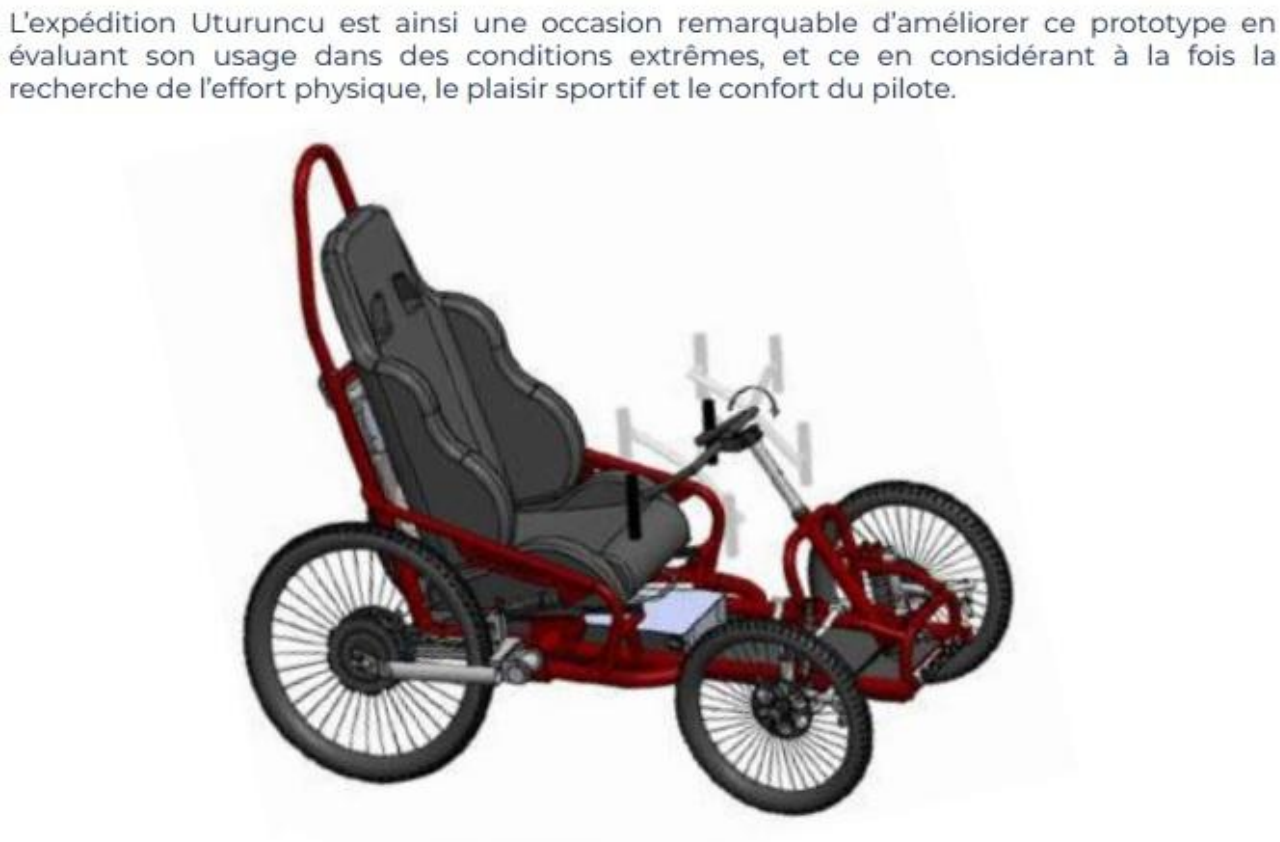

Plaquette Expédition Uturuncu - page 17

Sportifs et aventuriers avant tout, les handisportifs ont insisté notamment pour qu'en aucune manière le terrain ne soit adapté, spécifiquement à leur usage (un «déblayage " de l'accès à l'Uturuncu ayant été envisagé dans la première version de l'expédition !) : à l'équipe (handis comme valides) de s'adapter pour trouver des solutions de franchissement. Cet aspect intéresse particulièrement les écologues associés au projet, les milieux traversés étant parfois très sensibles (les handisportifs s'étant d'ailleurs déclarés intéressés par une sensibilisation à leur qualité écologique). De plus, tout en maintenant les moyens de sécurité et de santé indispensables aux athlètes handis, la volonté de tous est de ne pas aller au-delà des besoins essentiels : pas de véhicules plus que nécessaire, pas de cohorte de porteurs, couchage sous tente, nourriture suffisante mais sans surévaluation des besoins (moins de volumes à transporter, moins de déchets...). De ce point de vue, une expédition handisportive, du fait de la sobriété recherchée, parait moins impactante qu'un voyage handisportif «soft », à visée touristique classique (tel que le prévoyait le projet initial) où la recherche de confort prime fortement. C'est un bon point pour ce type de projets quant à leur moindre impact écologique dans l'univers global du voyage-aventure.

\section{CONCLUSION}


L'enjeu du projet d'expédition Uturuncu prône une nouvelle approche qui consiste à renverser les représentations sur le handicap, vers une «normalisation » (Marcellini, 2005), en proposant une logique où l'handisportif est lui-même leader et acteur de l'effort. Il s'agit d'une expédition où il n'y a plus les «aidants» et les «aidés », mais une équipe handisportifs/valides égaux et solidaires dans l'effort. Cette solidarité se retrouvant également dans les moments de rassemblement (pauses, contemplation de la nature, rencontres locales, etc.). La sportivité demandée n'éloigne pas pour autant le projet Uturuncu d'une approche écoresponsable : l'accent mis sur la recherche de performance nécessite de penser un usage minimal des aides modernes, donc un moindre impact. Le prototype de FTT à maindalier aidera ainsi seulement lors de passages difficiles et pas plus. L'affichage éco-responsable doit cependant rester modeste dans le cadre d'une expédition lointaine, donc par nature impactante en moyens de transports transcontinentaux. Il s'agira surtout de montrer une responsabilisation au jour le jour, la réduction de l'impact autant que faire se peut (appuyée sur des indicateurs fiables), voire la compensation (par soutien à des associations environnementales locales par exemple) et in fine la cohérence avec l'engagement handicapable, qui restera la valeur dominante du projet auprès des parties prenantes.

\section{Notes}

i «En Bref»-Handisport Magazine, 126(1), p. 37 - 2006

ii «L'événement X trême Day handisport »- Handisport Magazine, 96(1), p.10 - 1998

iii «L'aventure couleur sable » - Handisport Magazine, 124(1), pp. 42-43 - 2006

${ }^{\text {iv }}$ La Coccinelle, pensée par l'association Coccinelle-Handi (Gilbert Marmey président, Jean-Marc Fontvieille trésorier) est le ler FTT à 4 roues motrices et propulsion électrique (4 moteurs de 750 watts et 5 batteries de 16 $\mathrm{Ah})$ : avec une autonomie de $100 \mathrm{~km}$ à plat pou rune vitesse de $25 \mathrm{~km} / \mathrm{h}$ max et de $2000 \mathrm{~m}$ de dénivelé positif, elle peut grimper des pentes de $35 \%$.

v Le Boma possède 2 suspensions indépendantes à l'arrière, un bloc moteur actionnant les roues, 2 batteries NiMH 24 volts d'une autonomie de 15 a $20 \mathrm{~km}$, une vitesse max de $11 \mathrm{~km} / \mathrm{h}$ et se démonte en 6 parties pour potentiellement tenir dans un coffre de voiture. Cet engin propose des options qui en font le 1er FTT électrique à offrir le pilotage par Joystick et, entièrement pilotable avec la tête.

${ }^{\text {vi }}$ Le Mobile Dream est une amélioration de la Coccinelle avec une autonomie de $85 \mathrm{~km}$ (sur plat), une vitesse de $15 \mathrm{~km} / \mathrm{h}$ maximum. Il est homologué par le CERAH (Centre d'Étude et de Recherche de l'Appareillage de l'Handicap).

${ }^{\text {vii }}$ Le e-buggy, dérivé du "Buggy-bike, propose une autonomie d'environ $800 \mathrm{~m}$ positifs et de $25 \mathrm{~km}$. Cette autonomie peut être doublée par le rajout d'une batterie. Muni d'une marche arrière, il permet 4 modes de conduite programmables : chemins, sport, loisir et enfant.

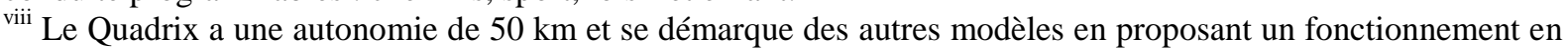
roue libre en descente, comme un FTT classique.

ix Alpiniste français amputé d'une jambe, 20 années d'expéditions à son actif avec l'ASVF (Association Sportive de Villefontaine).

${ }^{\mathrm{x}}$ Le maindalier est un pédalier à mains. Lorsqu'il complété d'une assistance électrique, il permet d'aider la personne à l'effort et au franchissement de certains obstacles.

\section{BIBLIOGRAPHIE / REFERENCES}

ANDRIEU B. (2017). Se fondre dans la nature - Figures de la cosmose - Cosmotique 1, Essai (broché).

BERTAUX D. (1997). Les récits de vie, 128, Nathan.

BOURDEAU P. (1994). Tourisme d'aventure : la traversée des apparences. Téoros, 13 (3), 610. 
CORNELOUP J. (2011). « La forme transmoderne des pratiques récréatives de nature. » Développement durable et territoires. Économie, géographie, politique, droit, sociologie 2.3.

ISSANCHOU D. \& PERERA E. (2020). Corps, Sport et Handicaps. Expériences et expérimentation sociales de la technologie (Tome 3), L'harmattan, coll. 《 Téraèdre », $208 \mathrm{p}$.

LE ROUX N., GALY A. et PERERA E. (2018). L'émergence du Fauteuil Tout Terrain en France entre bricolages et innovations : le rôle des pionniers de l'activité, Les carnets du Labex ITEM, Innovation et territoire de montagne.

https://labexitem.hypotheses.org/680

MARSAC A., LEBRUN A-M., \& BOUCHET P. (2012). Tourisme durable et expériences touristiques : un dilemme. Proposition d'un dispositif d'analyse appliqué à l'itinérance en milieu rural. Management \& Avenir, 56(6), 134.

MARCELLINI A. (2005). Des vies en fauteuil...: usages du sport dans les processus de déstigmatisation et d'intégration sociale. Paris : CTNERHI.

PANTALEON N. \& REICHHART F. (2017). «Expériences et perceptions des pratiquants de fauteuil de randonnée mono roue », Nature et Récréation, 31-40.

PERERA E. \& BELDAME Y. (2016). In Situ : interactions, situations et récits d'enquête. L'Harmattan, coll. «Mouvement des Savoirs », 302 p.

PERERA E., VILLOING G., RUFFIE S. \& GOSSET S., (2017). Le Fauteuil Tout Terrain, une "paire de chaussures de montagne»: expériences corporelles et reconfigurations identitaires, Science \& Motricité. https://doi.org/10.1051/sm/2017013

PERERA E., VILLOING G. et GALY A. (2020). «L'inventivité des alpinistes en fauteuil tout terrain : l'usage de la technologie au service de l'autonomie et de la mise en scène de la différence handi-capable », revue Nature \& Récréation, 41-51. https://www.naturerecreation.org/8-nat-et-recreation

RICHARD R. (2017). Approche socio-phénoménologique du corps sportif en situation de handicap, Etre footballeur en fauteuil, L'harmattan.

SIROST O. (2010). Le sens paysager. Sociétés, (3), 5-10.

VILLOING G., PERERA E. et LE ROUX N. (2017). The institutionalization of off-road wheelchair riding in France (1990-2015) : truly a sport of sharing and diversity », Sport in society, 1-14. 


\title{
The eco-friendly values of expeditions in all-terrain wheelchairs, a means to strengthen the visibility of the handi-capable difference.
}

\author{
ERIC PERERA, ROLAND THALER, ANDRE GALY
}

\section{Introduction}

Over the last few years, adventures in natural settings have become possible for disabled people (Pantaléon and Reichhart, 2017) through the use of ever more sophisticated contraptions, such as All-Terrain Wheelchairs (ATWs) (Villoing et al., 2017). ATWs procure social experiences (Issanchou and Perera, 2020) as well as new sensations (Perera et al., 2017), comparable to mountain biking. Its practice also provides the opportunity for mixing socially (able-bodied and disabled athletes).

On the face of it, ATW seems to pertain to a form of « contemplative tourism », instigating an « intimate sensory communion with nature and culture » (Bourdeau, 1994). It also closely relates to contemporary ethical and environmental concerns, with close links to so-called slow tourism (Symposium Slow tourism /Slow sport 2018 - University Littoral Côte d'Opale), by integrating movements from slow sports while communing with nature through cosmose (Andrieu, 2017). The tourist's footprint is thus minimised, their behaviours having been conceived in such a way as for them to commune with what they observe (Corneloup, 2011).

However, the sustainable dimension of ATWs raises questions, notably on the topic of the carbon impact of trips or concerning the use of motorised vehicles for logistics. In addition, new ATWs resort to electric assistance in order to autonomously roam the mountains (Perera et al., 2020). In this case, to what extent is promoting sustainable-oriented considerations still conceivable so as to overcome the idea of simply rendering "greener » (Marsac et al., 2012) the image of a far-off expedition in ATWs?

This question is all the more prominent due to the new generation of ATW athletes promoting handi-capacity by way of seeking a form of autonomy as complete as possible. These new approaches allow for the development of innovative hand-crank ATWs with electric assistance that might relegate eco-friendly concerns to a more secondary place.

\section{METHOD}

For this work, we examined the environmental dimension of ATW expeditions through interviews of a « life story » type (Bertaux, 1997), offering a socio-historical outlook on their organisation (interviews with Jean-François Porret (JFP) and Gilles Bouchet (GB), pioneers of this type of activity). Taking into account documents concerning the equipment of expeditions provided additional information. Interviews were also carried out with Gilbert Marmey (GM), the designer of an electric ATW, and Vincent Bourry (VB), a regular practitioner who is part of an innovating electric ATW project. This data was completed by an ethnographic study following an ATW expedition project to Chile, initiated in March 2018. The goal of this expedition is double: completing a trek integrating the ascent of the Uturuncu (6008 metres), and promoting a positive image of disability (or of the « otherwise capable » according to the expedition's sponsor Philippe Croizon, a famous swimmer amputated of all four limbs). The project was rescheduled to a yet unfixed date due to COVID 19. The data produced in situ (Perera and Beldame, 2016) by the researchers, authors of the present article and active accompanying members, concerns to this day observations pertaining to the logistical preparation of the expedition, as well as the training programme for it. This provides 
an insight into the social processes underlying the engagement of six people with diverse forms of disability using adapted vehicles, as well as nine accompanying members. In this manner, we could observe that the promotion of the expedition favoured the autonomy of the handi-athletes while, on the face of it, moving further away from eco-friendly concerns. Indeed, the expected sportiness, guaranteeing a handi-capable promotion, involves the use of electric assistance and, more than anything, the support of heavy logistics (4-wheel drives, power generators, etc.). This does not allow, such as it is, a sufficiently effective environmentoriented display, in spite of the true desire of everyone involved to limit impacts. It does however provide the opportunity to identify the paths for progressing toward an « ecoaccountability » of the group during the preparation and the actual expedition (using optimised vehicles, condensing itineraries, sleeping in tents ...).

\section{THE BEGINNING OF ATW IN FRANCE, PRINCIPLES OF SHARING AND PROXIMITY TO NATURE}

ATW practices were initiated in France in 1990 by JFP, a renowned French alpinist who became quadriplegic after a paragliding accident in 1989. During re-education, he discovered the "Cobra", the first ATW to be inspired by mountain-bike technology. On the strength of his mountaineering experience, he completed a first expedition to Kula Kangri (Tibet) in 1993, climbing to an altitude of $5600 \mathrm{~m}$. In this way, he rekindled «this pleasure of making projects, of having dreams [...], of feeling the uncertainty of success [...] it really was a strong component for continuing to live ». With the help of GB, they tested a hundred itineraries, mainly in the Alps (www.ftt.free.fr). The dynamics of this ATW practice were intentionally «turned towards sportive hiking and the quest for autonomy out in nature » (HandiMag, Porret, 1999, p. 41). By the end of the years 2000, an accessible offer for ATWs was finally developed (Villoing et al., 2017). Whether through hikes or expeditions, this ATW pioneer regained access to the mountain, as well as to values such as sharing and social mixing between disabled/non-disabled individuals. This notion of sharing, just like the one which consists in instigating a certain proximity to nature, constitute the foundation for ATW practice and bring it closer, through its slow sport dimension and its conception of travelling, to slow tourism.

\subsection{ATW practices: «the mountain of sharing » more than performance}

The expeditions led by JFP were, first and foremost, social experiences that played a central role in the continuity of his practice of alpinism, going from a quest for performance towards a sharing dynamic. "There really was before (the accident), the mountain of performance and after, the mountain of sharing [...] that doesn't mean that there was no sharing before and that there is no performance after $»$. It was mainly thanks to this transition towards a sharing mind-frame that he was able to go back to organising «expeditions like before ». He made sure, however, that «those who came with me experienced their own pleasure [...] For myself, I necessarily have the aim of [...] feeling again the sensations of an expedition in my legs [...] (with) a level of discomfort that forces me to soldier on like I did before, a level of discomfort which is finally quite similar... ». JFP noted, within his personal $\log$, the importance of the group: the adventure «would not have been possible without the constant care of the entire team and its capacity to adapt to the constraints of the ATW » (Minya Kongka expedition, China, 1995). In this manner, he highlighted the effects of mixing disabled and non-disabled people, allowing for a strong social experience. 
What is more, his condition as quadriplegic produced another relationship with local populations during his expeditions. Indeed, the ATW gave the opportunity to get closer to populations which brought «so much kindness in all these beautiful encounters 》 (Minya Kongka logbook, 1995). Thus, the expeditions led by JFP evolved into an «adventure, not in the sense of adventurous, but in the sense of making contact with people, feeling this extraordinary human warmth. » This was a new way of viewing expeditions, advocating « $a$ mountain of sharing rather than a mountain of performances ».

JFP favoured social links (teammates, local populations), while the impulse for sharing took over the search for performance. Practicing ATW thus became the opportunity to discover natural spaces sportively and in the company of peers (Sirost, 2010), generating a different relationship with nature.

\subsection{ATW practice, a mountain-friendly activity}

The environment plays a central role in ATW expeditions. Formerly, JFP used to seek exploits: opening a new path in Peru, accessing an untouched summit in Pakistan... After his accident, however, he continued to venture out into far off and wild places, his sensations seemingly multiplied by his new condition: «the weather is clearing and offering us a superb view of the entire Kula Kangri range. I can see materialise under my own eyes what I had imagined from photos back in 87 [...] We can see the whole itinerary of the Japanese path and we clearly dominate the glacier accessing alpinism as seen from a wheelchair ... [...]. Only too rare moments of happiness are these minutes of total freedom, lived intensely at the other end of the world». This approach comes with a deep respect for the mountain and took on all the more importance after his accident: "We don't touch nature [...] we foresee the adaptations which will allow disability to access this untouched nature. Rather than bring nature into the grasp of disability, we bring disability into the reach of nature ».

ATWs are conceived in order to facilitate access to full-nature hikes. For JFP, using this contraption, between «overcoming limits» and «the happiness of autonomy », provides evidence that disability is not an obstacle to adventure. Whether before or after his accident, the means employed remained the same (aeroplanes, vehicles, bearers/animals). His quadriplegia, however, obliged him to go everywhere with his ATW, his standard wheelchair, and a large amount of equipment specific to his condition. This induced much excess weight compared to «normal» conditions. What is more, he required external help in order to complete the ascent right up to the summit (extra bearers, and more than anything, traction by animals...).

Other than these minimum requirements, the importance of the environment was such for JFP that he considered that it was disability that should adapt to the mountain. ATWs represent a true sporting instrument (Richard, 2017) for high mountain areas, allowing JFP to complete many expeditions.

\section{REPRESENTATIONS AND LEVERS OF A SUSTAINABLE EXPEDITION IN ATW}

As pioneers of ATW in France, JFP and GB opened the path for the possibility of gaining access to the most extreme natural spaces. This inspired the development of its practice, largely facilitated by the French Federation for Handisport $(\mathrm{FFH})^{\mathrm{x}}$, in order to provide an answer to the public's keen interest in outdoor sports. With this in mind, the FFH created, as early as $1998^{\mathrm{x}}$, several cultural events, such as the «X Treme Day Handisport » in the Aveyron (Southern French Department) for example. On a federal level, adventure sports 
flourished while strongly highlighting expeditions. The FFH thus «appropriated» these activities, which became a complement to the competitive dimension that had dominated up until then. The funding provided for expeditions and raids ${ }^{x}$ profited to the development of ATW use in general.

Simultaneously, the quest for autonomy in order to overcome natural, but also social, barriers generated the development of ATWs with electric assistance. With electric ATWs, "we change the paradigm » in order to achieve «complete autonomy », according to Vincent Boury (Le Roux et al., 2018). Although this newfound autonomy works both for the descent and the ascent, this new approach disrupted the abled/disabled relations existing within classic ATW practice and, all the more so, because the new generation seek an even more sporting type of engagement. This technology was conceived above all as an autonomous sporting engagement and thus deviates from a sustainable outlook.

\subsection{Electric assistance, a help for full-nature sportivity}

The first models of electric assisted ATWs appeared in 2006, enabling the descent of mountainous paths, but also and foremost, facilitating ascents without other assistance. With the advent of the Coccinelle ${ }^{\mathrm{x}}$ [Ladybird], developed by the Coccinelle-Handi association and the Boma ${ }^{\mathrm{x}}$, industrialised by the English company Molten Rock, these contraptions were an evolution within ATW practice, granting the disabled person increasingly more autonomy.

Other models of AE ATWs such as the Mobile Dream ${ }^{\mathrm{x}}$, the $e$-buggy ${ }^{x}$ or the Quadrix also came onto the market, simultaneously sturdy and endowed with great capacities for autonomy thanks to the evolution of batteries. However, these EA ATWs were conceived rather more for "rambling" (VB) than for accomplishing sporting performances or expeditions such as the classic ATWs allow, which can easily be tinkered with as did, for instance, the head of the expedition, Gérard Genthon (GG) ${ }^{x}$. In this context, the EA ATW opened new ways of enjoying full-nature environments, centred on a family leisure practice and not performance.

Nevertheless, it was truly the EA ATW that the new generation of handiathletes chose for the Uturuncu expedition: a choice of speed and physical participation, running up against the «rambling » spirit, leading to a project for a new type of ATW with an electricallyassisted hand-crank ${ }^{\mathrm{x}}$, allowing the pilot to play an active part in their activity.

The values carried by a part of the team, both handiathletes and non-disabled members, favouring autonomy rather than sportivity, led to a fracture which induced a change of expedition leader, passing from GG to Emmanuel Senin, from the old generation to the new one. The rearranged team thus met up on $9^{\text {th }}$ August 2019 in order to refocus the expedition on the stakes and considerations of the handiathletes, including the new ATW contraption with an EA hand-crank, but also a more sporty expedition itinerary. The choice of making the handiathlete a main actor of the ascent effort went through the use of adapted technologies and an itinerary with a stronger sporting and risk-taking dimension. However, this search for sporting autonomy, so different from the spirit of the initial expedition (named Handi'Cap Cordillière), did not prevent the team from supporting values such as handiathlete/non-disabled sharing and upholding a certain level of communion with nature.

\subsection{A sustainable positioning of ATW expeditions}

The common values of the Handi'Cap Cordillière expedition were the subject of debates during the training weekend in the Luberon's mountain on $6^{\text {th }}$ June 2019 . The issue was to collectively find the image that the team wanted to give the expedition in order to 
exhibit a united front before the parties involved. This time for exchange turned out to be essential, since the vision of the Handi'Cap Cordillière expedition seemed to strongly differ depending on the team members. For some, the most important was the group's cohesion, for others the central question was communication in order to find partnerships and sponsors. What needed to be highlighted in order to reflect the spirit and values of the expedition? This was the heart of the discussions and provided a rich and decisive debate for the future of the project.

Emmanuel thus put forward the importance of the sporting and humanitarian values in relation to the sponsors who had been contacted: "I had difficulty identifying with the support (speaking of the pre-existing brochure). [...] I think it would be interesting to revise what we put forward: the prowess side, the 6000 metres... [...] if we choose foundations ...I would also talk about the humanitarian side [...] it's an extra for the people we can help on-site, and it opens more doors ».

The choice of these values, approved by the team, alongside the difficulty experienced by Emmanuel to identify with the communication methods in use at the time, led to a change in orientation for the expedition. Indeed, the «slow » values carried up until then, notably by GG, were not really compatible with a showcasing of handi-athlete performance and autonomy, under cover of a form of solidarity between disabled/abled surrounding a classical ATW (Perera et al., 2020).

From this situation stemmed a reorganised team and the birth of a new project for the expedition, from then onward named "Uturuncu". This project was marked by a wish to promote, above all else, the handi-capable dimension, all the while integrating eco-friendly principles. This topic had indeed been the subject of numerous debates, with for instance the question of using vehicles in situ: "[...] in the initial project, there were nine $4 X 4 s !$ [...] At this point, aren't we more in a situation of comfort rather than an expedition? And this clashes with the eco-friendly dimension. Can we not pack in tighter together and then we'd only need 3? ». Another topic of discussion was eating: "Eating local food seems selfevident, but it's complicated, what do we eat? [...] Maybe we can discuss and find some sort of balance ». Ultimately, a need was revealed to collectively define the eco-friendly lines to be pursued during the expedition: "We had not defined amongst ourselves the levers to make the project eco-friendly and therefore to give us credibility when we speak of it. » (Julien).

Searching for a balance between a sporting approach and an eco-friendly one thus guided the reflection and helped determine not only the itinerary of the new Uturuncu project, but also the conception of the new ATW with EA hand-crank that was to be tested. 


\section{UNE EXPÉRIENCE AU BÉNÉFICE DE TOUS}

Au-delà de la performance sportive, notre expédition revêt des enjeux sociétaux d'aujourd'hui et de demain : promotion de la solidarité, partage de savoirs et savoir-faire avec les acteurs locaux, développement de l'accessibilité aux sports et à la nature pour tous...

tels sont les retours d'expérience attendus de cette aventure collective.

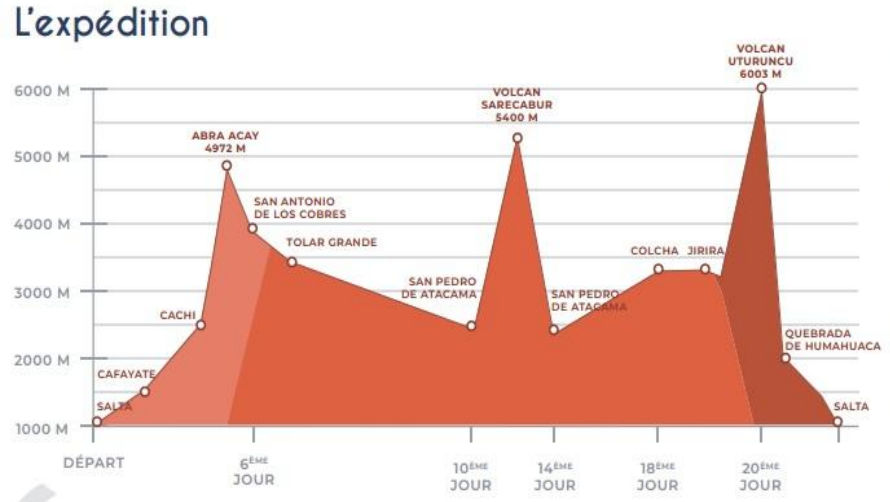

Uturuncu Expedition Brochure - page 6
3) LES SITES INCONTOURNABLES

Cafayate : Située dans une zone très aride, elle est considérée comme la plus belle région d’Argentine.

Salar d'Uyuni : La plus grande étendue de sel du monde. Ce paysage désertique d'un blanc éclatant, cactus.

Désert d'Atacama : Connu pour être la région la plus aride sur Terre. Certains secteurs peuvent être prives de precipitations pendant 50 ans.

Quebrada de Humahuaca : Profond canyon déclaré Patrimoine Culturel et Naturel de l'Humanité

RN 40 : Le plus haut col (Abra Acay $4895 \mathrm{~m}$ ) de la mythique Ruta 40 qui traverse la cordillère des

The new promotional brochure highlights the differences in altitude which the team members will have to face and the efforts which the handiathletes will have to make in order to complete the various ascents autonomously. These new challenges led to the development of innovative devices, including the ATW with hand-cranks that are electrically assisted (see image below), for an autonomous engagement as well as a sportier one. In addition to the summits to attain, of which the Uturuncu and its $6003 \mathrm{~m}$, that are highlighted, the itinerary also points out the «key sites » which the expedition will go through. The contemplative dimension of the expedition thus remains, in spite of an increased sportivity in the itinerary.
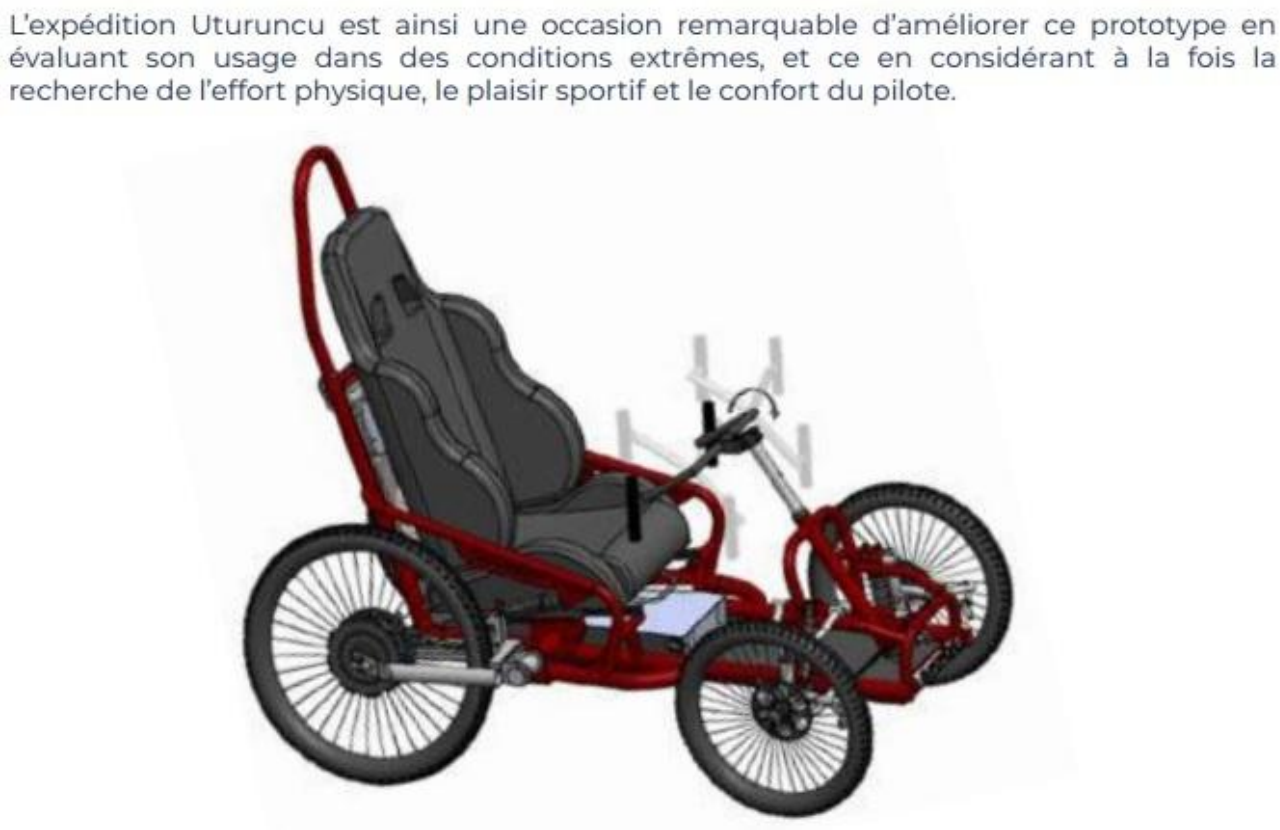

Uturuncu Expedition Brochure - page 17 
As athletes and adventurers above all, the handiathletes notably insisted that the terrain be in no way specifically adapted for their use (a «clearing out» of the access to the Uturuncu having been envisaged in the first version of the expedition project!): it is up to the team (disabled and non-disabled) to adapt and find solutions for getting past obstacles. This aspect is particularly interesting for the environmental engineers associated to the project, since the areas the team will go through can occasionally be very sensitive (the handiathletes having indeed expressed the desire to learn more about ecological qualities). Additionally, while maintaining the security and healthcare means that are indispensable to handiathletes, the wish of every member of the team is to not go beyond essential necessities: no unnecessary extra vehicles, no hoard of bearers, sleeping under tents, sufficient food but nothing beyond basic needs (thus less volume to transport, less waste ...). From this point of view, a handisportive expedition, because of the imposed sobriety, appears as having less of an impact than a «soft » handisportive trip, with a classic touristic goal (as the initial project was aiming for), where the question of comfort strongly predominates. This is a strong point for this type of project with a diminished ecological impact within the global universe of adventure travelling.

\section{CONCLUSION}

The Uturuncu expedition project advocates a new approach which consists in turning around the representations of disability, going towards a «normalisation » (Marcellini, 2005), by proposing a perspective in which the handiathlete is both the leader and the actor of their effort. This is an expedition where there are no longer the «helpers » and those who are « helped », but a team of disabled/non-disabled individuals who are equal and interdependent in the effort required. This form of solidarity can also be found during moments of assembly (pauses, contemplation of nature, meetings with the locals, etc.). The demanded sportivity does not distance the Uturuncu from an eco-friendly approach: the accent put on the quest for performance implies a minimal use of modern assistance means, and thus a limited impact. The hand-crank ATW prototype will therefore only help to get past difficult spots, and no more than that. The eco-friendly label must however be used moderately in the context of a long-distance expedition, which will, by nature, have a certain impact in terms of transcontinental transport means. The goal will be, first of all, to show just how, day-to-day responsibility and the strictest possible impact minimisation (using reliable indicators), maybe even to the extent of compensation (by supporting local eco-friendly associations for instance), and in fine coherence with the handi-capable engagement, which will remain the dominant value of the project for all the parties involved.

\section{Notes}

${ }^{\mathrm{x}}$ «En Bref »- Handisport Magazine, 126(1), p. 37 - 2006

x «L'événement X trême Day handisport »-Handisport Magazine, 96(1), p.10 - 1998

${ }^{x}$ «L'aventure couleur sable »- Handisport Magazine, 124(1), pp. 42-43 - 2006

$\mathrm{x}$ The Coccinelle, developed by the Coccinelle-Handi association (Gilbert Marmey president, Jean-Marc Fontvieille treasurer) was the first electric four-wheel drive ATW (4 motors of 750 watts and 5 batteries of 16 $\mathrm{Ah}$ ): with an autonomy of $100 \mathrm{~km}$ on level ground, for a top speed of $25 \mathrm{~km} / \mathrm{h}$ and $2000 \mathrm{~m}$ of positive elevation, it can climb $35 \%$ inclines.

${ }^{\mathrm{x}}$ The Boma disposes of 2 independent suspensions at the back, a engine block operating the wheels, 2 NiMH batteries of 24 volts with an autonomy range of 15 to $20 \mathrm{~km}$, a top speed of $11 \mathrm{~km} / \mathrm{h}$, and can be dismantled into 
6 parts in order to potentially fit in a car boot. This contraption offers options which make it the first ATW to provide joystick piloting and can be entirely piloted with the head.

${ }^{x}$ The Mobile Dream is an improvement on the Coccinelle with an $85 \mathrm{~km}$ autonomy range (on level ground), a top-speed of $15 \mathrm{~km} / \mathrm{h}$. It is certified by the CERAH (Study and Research Centre for the Equipment of Disability).

${ }^{x}$ The e-buggy, derived from the "Buggy-bike, offers an autonomy range of around $800 \mathrm{~m}$ positive ascent and 25 $\mathrm{km}$. This autonomy can be doubled by adding an extra battery. Equipped with a reversing option, it offers 4 programmable driving options: paths, sport, leisure, child.

${ }^{\mathrm{x}}$ The Quadrix has a $50 \mathrm{~km}$ autonomy range and differs from the other models by offering a free-wheeling option on downslopes, like a classic ATW.

${ }^{\mathrm{x}}$ French alpinist who was amputated of one leg, 20 years of experience in the field of expeditions with the ASVF (Villefontaine Sporting Association).

${ }^{\mathrm{x}} \mathrm{A}$ hand-crank is a pedaling system operated by hand. When it is completed with electric assistance, it can help with the pilot's effort as well as for passing certain obstacles.

\section{REFERENCES}

go to Bibliographie at the end of French version. 